\title{
A autopercepção da voz do adolescente
}

\author{
Adolescents'self-perception of voice
}

\author{
Anna Alice Figueirêdo de Almeida ${ }^{1}$, Mara Behlau²
}

\begin{abstract}
RESUMO
Objetivo: Verificar a autopercepção dos adolescentes em relação à própria voz. Métodos: Aplicou-se um questionário com perguntas relacionadas à voz e comunicação a 80 adolescentes voluntários, de 10 a 19 anos, 58 (72,5\%) do gênero feminino e 22 (27,5\%) do masculino, participantes do Programa de Saúde Integral do Adolescente do Estado de São Paulo. Os próprios estudantes responderam ao questionário, cujos dados foram analisados estatisticamente. Resultados: A faixa etária predominante dos voluntários foi de 16-17 anos (23-28,8\%). Metade deles (41 - 51,3\%) atribuiu características positivas a sua voz, classificando-a como normal (42 $-52,5 \%)$; muitos $(33-41,3 \%)$ não perceberam nenhuma modificação vocal na adolescência; a maioria demonstrou satisfação com sua emissão $(65-81,3 \%)$, referindo que sua voz combina com a personalidade $(27$ - 33,8\%) e, portanto, não desejava modificá-la $(61-76,3 \%)$. Os três hábitos mais apontados como nocivos à voz foram: gritar (30 - 37,5\%), tomar bebida gelada $(26-32,5 \%) \mathrm{e}$ tabagismo (22 - 27,5\%); por sua vez, foram mencionados como hábitos benéficos: beber água (31 - 38,8\%), cantar (30 - 37,5\%) e não gritar $(20-25 \%)$. Os rapazes referiram problemas vocais relacionados ao pitch $(54,5 \%, \mathrm{p}<0,001)$ e as moças, em relação a loudness $(32,1 \%, \mathrm{p}<0,001)$, apontados como fatores de comprometimento na comunicação. Conclusão: A percepção do adolescente sobre a sua voz e comunicação interfere na sua interação com o outro, no seu ato de pensar, agir, fazer e na sua articulação com a realidade e a sociedade em que vive.
\end{abstract}

Descritores: Comunicação; Voz; Distúrbios da voz; Adolescente; Auto-imagem

\section{INTRODUÇÃO}

A adolescência é a fase dos 10 aos 19 anos de idade, baseada no aparecimento inicial das características sexuais secundárias, pelo desenvolvimento de processos psicológicos e de padrões de identificação que evoluem da fase infantil para a adulta, e pela transição de um estado de dependência para outro de relativa autonomia ${ }^{(1)}$.

A muda vocal é apenas um aspecto das alterações que ocorrem nessa fase; todavia obtém destaque, pois é a partir da sua voz que o adolescente poderá se comunicar, se expressar, se desenvolver em seu meio social. Assim, acredita-se que a intervenção fonoaudiológica realizada com adolescentes deve ser específica e endereçada a essa faixa etária, priorizando todas as particularidades que lhes são inerentes ${ }^{(2-3)}$.

Trabalho vinculado ao Centro de Estudos da Voz - CEV - São Paulo (SP), Brasil.

(1) Pós-graduanda em Ciências pelo Departamento de Psicobiologia da Universidade Federal de São Paulo - UNIFESP - São Paulo (SP), Brasil; Professora assistente do Curso de Fonoaudiologia da Universidade Federal da Paraíba - UFPB - João Pessoa (PB), Brasil.

(2) Doutora; Professora colaboradora do Programa de Pós-graduação em Distúrbios da Comunicação Humana da Universidade Federal de São Paulo - UNIFESP - São Paulo (SP), Brasil; Diretora do Centro de Estudos da Voz - CEV - São Paulo (SP), Brasil.

Endereço para correspondência: Anna Alice Figueirêdo de Almeida. R. Alves Guimarães, 408/61, Jardim América, São Paulo - SP , CEP: 05410-000.

E-mail: anna_alice@uol.com.br

Recebido em: 1/4/2008; Aceito em: 7/9/2008
Atualmente, as pesquisas abordam com frequência a autoavaliação ou autopercepção vocal, pois se sabe que a partir dela pode-se captar a opinião do paciente em relação a sua voz, tornando-se mais um parâmetro a ser enfatizado durante a avaliação. Esses conhecimentos podem auxiliar em ações junto ao indivíduo disfônico, uma vez que a autopercepção e a psicodinâmica vocal são fatores importantes em um processo terapêutico ${ }^{(4)}$.

Em um estudo realizado com 400 indivíduos do sexo masculino a fim de investigar a memória da muda vocal ocorrida durante a adolescência ${ }^{(5)}$, observaram que a maioria $(78,8 \%)$ notou mudanças na voz durante a puberdade; $25 \%$ dos entrevistados relataram que a muda vocal ocorreu aos 14 anos; 22,5\% disseram que a mesma durou de 12 a 24 meses. As principais características vocais relatadas foram relacionadas ao pitch, à qualidade vocal e a loudness. Os sentimentos e comportamentos referidos em função da alteração vocal durante a muda foram negativos. Assim, concluíram que os homens se lembram da muda vocal fisiológica e que não há sentimentos negativos relacionados às alterações vocais típicas daquele período.

Em uma pesquisa sobre uma intervenção fonoaudiológica com adolescentes ${ }^{(6)}$, constataram que os hábitos nocivos à voz mais citados antes da intervenção foram: gritar $(49,4 \%)$, falar muito $(29,8 \%)$ e ingestão de líquido gelado $(25,6 \%)$. Em contrapartida, os hábitos benéficos à voz foram: beber água e em temperatura ambiente $(46,1 \%)$, não gritar $(26,0 \%)$ e não 
falar muito (25\%). Quanto aos dados sobre os termos descritivos atribuídos a sua própria voz, percebeu-se que $75,8 \%$ dos adolescentes atribuíram termos positivos a sua voz, porém foi estatisticamente significante que os adolescentes do sexo masculino citaram mais termos negativos, mais especificamente, relacionados à instabilidade e quebras da frequência vocal.

Ter acesso aos pontos de vista e perspectivas de adolescentes é um ponto central para a criação e organização de programas e intervenções que visem promover à saúde dos $\operatorname{mesmos}^{(7)}$.

Assim, o objetivo deste estudo foi verificar a autopercepção dos adolescentes em relação à própria voz, a fim de posteriormente, elaborar uma intervenção mais específica voltada a essa população.

\section{MÉTODOS}

Esta pesquisa foi avaliada e aprovada pelo Comitê de Ética e Pesquisa do Centro de Estudos da Voz - CEV, protocolo $\mathrm{n}^{\circ} 0411 / 03$.

Participaram desta pesquisa 80 adolescentes pertencentes ao Programa de Saúde Integral do Adolescente da Secretaria de Saúde do Estado de São Paulo, sendo 8 (72,5\%) do gênero feminino e $22(27,5 \%)$ do masculino, com a faixa etária de 10 a 19 anos, fase em que compreende a adolescência ${ }^{(1)} \mathrm{e}$ com o nível escolar compreendido entre ensino fundamental incompleto e nível superior incompleto (Tabela 1).

A coleta dos dados teve como base o questionário utilizado no Programa de Saúde Integral do Adolescente da Secretaria de Saúde do Estado de São Paulo, que tem como objetivo conhecer o perfil da comunicação e da voz dos adolescentes que o frequentam, que foi modificado para uso nesta pesquisa. O protocolo utilizado é composto de questões do tipo múltipla escolha e descritivas (Anexo 1). Aborda, além de informações pessoais, as seguintes temáticas que foram divididas de forma sistemática em três blocos: o primeiro, que compreendia da pergunta 1 a 7 , era relacionado ao autoconhecimento, à autopercepção e à auto-imagem da voz, trazendo à luz questões referentes à muda vocal; o segundo, da pergunta 8 e 9, abordava

Tabela 1. Distribuição dos adolescentes participantes $(n=80)$ em relação ao sexo, faixa etária e grau de escolaridade

\begin{tabular}{lcc}
\hline Variável & $\mathrm{N}$ & $\%$ \\
\hline Sexo & & \\
$\quad$ Feminino & 58 & 72,5 \\
$\quad$ Masculino & 22 & 27,5 \\
\hline Faixa etária & & \\
$10-11$ anos & 06 & 7,5 \\
$12-13$ anos & 18 & 22,5 \\
$14-15$ anos & 21 & 26,2 \\
$16-17$ anos & 23 & 28,8 \\
$18-19$ anos & 12 & 15,0 \\
\hline Grau de escolaridade & & \\
Ensino Fundamental incompleto & 33 & 41,2 \\
Ensino Médio incompleto & 27 & 33,8 \\
Ensino Médio completo & 11 & 13,8 \\
Ensino Superior incompleto & 5 & 6,2 \\
$\quad$ Não respondeu & 4 & 5,0 \\
\hline
\end{tabular}

os hábitos vocais mais frequentes dos adolescentes e por fim, o terceiro, da pergunta 10 a 15, se referia à autopercepção do adolescente em relação à comunicação e à influência que a voz poderia surtir na mesma.

No período de maio e junho de 2005, a pesquisadora reuniu na sala de espera, adolescentes frequentadores da Casa do Adolescente no Centro de Saúde Pinheiros - uma das extensões do Programa de Saúde Integral do Adolescente - e explicitou o objetivo da pesquisa. Os interessados a participar da mesma assinaram o Termo Livre e Esclarecido e responderam às questões com a mediação da pesquisadora, que esclareceu as dúvidas quando solicitada.

Registrou-se em planilha específica as respostas aos questionários, representando cada participante; as respostas foram categorizadas e permitiram a formação de critérios e categorias para as questões descritivas. Essas categorias foram divididas em: opinião sobre a própria voz, autopercepção da voz, nível de satisfação vocal e autopercepção das modificações vocais.

Posteriormente, essa planilha eletrônica foi submetida à estatística descritiva e a uma inferência estatística necessária. O teste Qui-quadrado foi aplicado para as variáveis categóricas: sexo, faixa etária e autopercepção da voz, que foi o termo atribuído à forma que o adolescente descreve e o valor que ele designa a sua voz. Considerou-se o resultado significativo o valor de $\mathrm{p}<0,05$ ou $5 \%$.

\section{RESULTADOS}

A Tabela 2 apresenta a análise comparativa das categorias referentes à autopercepção da voz dos adolescentes em relação ao sexo.

A Tabela 3 apresenta a análise comparativa das categorias referentes à autopercepção da voz dos adolescentes em relação à faixa etária.

A Tabela 4 apresenta a distribuição numérica e percentual dos hábitos nocivos e benéficos à própria voz na opinião dos adolescentes.

\section{DISCUSSÃO}

O Programa de Saúde Integral do Adolescente da Secretaria de Saúde do Estado de São Paulo reúne profissionais de várias formações: médicos, psicólogos, fisioterapeutas, nutricionistas, assistentes sociais, fonoaudiólogos, entre outros. Cada um entende o adolescente sob um aspecto mais específico, contribuindo com uma parcela de seu conhecimento, o que possibilita uma visão mais ampla desses indivíduos, visando, assim, propiciar um atendimento mais completo aos mesmos.

Nesta pesquisa, a amostra foi composta por 80 adolescentes participantes do mencionado Programa, em sua maioria do sexo feminino (72,5\%), na faixa etária de $16-17$ anos $(28,8 \%)$ e ensino fundamental incompleto $(41,2 \%)$ como grau de escolaridade.

No tocante à comparação da opinião de adolescentes em relação a sua voz, a variável sexo (Tabela 2) apresentou uma diferença significante $(\mathrm{p}<0,001)$; observou-se que $14(63,7 \%)$ adolescentes do sexo masculino e 27 (46,5\%) do sexo feminino da amostra atribuíram qualidades positivas a sua voz; quando 
Tabela 2. Comparação da autopercepção da voz dos adolescentes $(n=80)$ em relação ao sexo

\begin{tabular}{|c|c|c|c|c|c|}
\hline \multirow{2}{*}{ Variáveis } & \multicolumn{2}{|c|}{ Masculino } & \multicolumn{2}{|c|}{ Feminino } & \multirow{2}{*}{ Valor de $\mathrm{p}$} \\
\hline & $\mathrm{N}$ & $\%$ & $\mathrm{~N}$ & $\%$ & \\
\hline \multicolumn{6}{|l|}{ Opinião sobre a própria voz } \\
\hline Qualidades positivas & 14 & 63,7 & 27 & 46,5 & \multirow{5}{*}{$0,001^{*}$} \\
\hline Qualidades negativas em relação ao pitch & 6 & 27,3 & 4 & 6,9 & \\
\hline Qualidades negativas em relação a loudness & 0 & 0 & 4 & 6,9 & \\
\hline Indiferente & 0 & 0 & 23 & 39,4 & \\
\hline Não respondeu & 2 & & 0 & 0 & \\
\hline \multicolumn{6}{|l|}{ Autopercepção da voz } \\
\hline Normal & 10 & 45,5 & 32 & 55,2 & \multirow{4}{*}{$0,001^{*}$} \\
\hline Desviada quanto ao pitch & 12 & 54,5 & 6 & 10,3 & \\
\hline Desviada quanto à loudness & 0 & 0 & 18 & 31,0 & \\
\hline Desviada quanto à qualidade vocal & 0 & 0 & 2 & 3,5 & \\
\hline \multicolumn{6}{|l|}{ Nível de satisfação vocal } \\
\hline Satisfeito(a) & 13 & 59,1 & 52 & 89,6 & \multirow{5}{*}{$0,001^{*}$} \\
\hline Característica pessoal & 13 & 100 & 40 & 76,9 & \\
\hline Características positivas & 0 & 0 & 12 & 23,1 & \\
\hline Insatisfeito(a) & 9 & 40,9 & 4 & 6,9 & \\
\hline Não respondeu & 0 & 0 & 2 & 3,5 & \\
\hline \multicolumn{6}{|l|}{ Autopercepção quanto às modificações vocais } \\
\hline Sim & 12 & 54,5 & 8 & 14,1 & \multirow{5}{*}{$0,001^{*}$} \\
\hline Pitch & 6 & 50,0 & 6 & 75,0 & \\
\hline Loudness & 6 & 50,0 & 2 & 25,0 & \\
\hline Não & 6 & 27,3 & 27 & 46,5 & \\
\hline Não lembra & 4 & 18,2 & 23 & 39,4 & \\
\hline Total & 22 & 100 & 58 & 100 & \\
\hline
\end{tabular}

Teste do Qui-quadrado; * Diferenças significantes $p<0,05$

Tabela 3. Comparação da autopercepção dà voz dos adolescentes $(n=80)$ em relação à faixa etária

\begin{tabular}{|c|c|c|c|c|c|c|c|c|c|c|c|}
\hline \multirow{2}{*}{ Variáveis } & \multicolumn{2}{|c|}{ 10-11 anos } & \multicolumn{2}{|c|}{$12-13$ anos } & \multicolumn{2}{|c|}{$14-15$ anos } & \multicolumn{2}{|c|}{$16-17$ anos } & \multicolumn{2}{|c|}{ 18-19 anos } & \multirow{2}{*}{$\begin{array}{l}\text { Valor } \\
\text { de } p\end{array}$} \\
\hline & $\mathrm{N}$ & $\%$ & $\mathrm{~N}$ & $\%$ & $\mathrm{~N}$ & $\%$ & $\mathrm{~N}$ & $\%$ & $\mathrm{~N}$ & $\%$ & \\
\hline \multicolumn{12}{|l|}{ Opinião sobre a própria voz } \\
\hline Qualidades positivas & 1 & 16,7 & 9 & 50,0 & 6 & 28,6 & 15 & 65,2 & 10 & 83,3 & \multirow{5}{*}{$0,004^{*}$} \\
\hline Qualidades negativas em relação ao pitch & 0 & 0 & 5 & 27,8 & 6 & 28,6 & 0 & 0 & 0 & 0 & \\
\hline Qualidades negativas em relação a loudness & 0 & 0 & 0 & 0 & 3 & 14,2 & 0 & 0 & 0 & 0 & \\
\hline Indiferente & 4 & 66,6 & 3 & 16,7 & 6 & 28,6 & 8 & 34,8 & 2 & 16,7 & \\
\hline Não respondeu & 1 & 16,7 & 1 & 5,5 & 0 & 0 & 0 & 0 & 0 & 0 & \\
\hline \multicolumn{12}{|l|}{ Autopercepção da voz } \\
\hline Normal & 6 & 100 & 9 & 50,0 & 8 & 38,1 & 13 & 56,5 & 6 & 50,0 & \multirow{4}{*}{$<0,001^{*}$} \\
\hline Desviada quanto ao pitch & 0 & 0 & 7 & 38,9 & 5 & 23,8 & 2 & 8,7 & 4 & 33,3 & \\
\hline Desviada quanto à loudness & 0 & 0 & 2 & 11,1 & 8 & 38,1 & 8 & 34,8 & 0 & 0 & \\
\hline Desviada quanto à qualidade vocal & 0 & 0 & 0 & 0 & 0 & 0 & 0 & 0 & 2 & 16,7 & \\
\hline \multicolumn{12}{|l|}{ Nível de satisfação vocal } \\
\hline Satisfeito(a) & 6 & 100 & 12 & 66,7 & 16 & 76,2 & 19 & 82,6 & 12 & 100 & \multirow{5}{*}{0,082} \\
\hline Característica pessoal & 4 & 66,6 & 12 & 100 & 12 & 75,0 & 11 & 57,9 & 8 & 66,7 & \\
\hline Características positivas & 2 & 33,4 & 0 & 0 & 4 & 25,0 & 8 & 42,1 & 4 & 33,3 & \\
\hline Insatisfeito(a) & 0 & 0 & 6 & 33,3 & 5 & 23,8 & 2 & 8,7 & 0 & 0 & \\
\hline Não respondeu & 0 & 0 & 0 & 0 & 0 & 0 & 2 & 8,7 & 0 & 0 & \\
\hline \multicolumn{12}{|l|}{ Autopercepção quanto às modificações vocais } \\
\hline Sim & 1 & 16,7 & 4 & 22,2 & 9 & 42,9 & 2 & 8,7 & 4 & 33,3 & \multirow{5}{*}{$0,003^{*}$} \\
\hline Pitch & 1 & 100 & 1 & 25,0 & 4 & 44,4 & 2 & 100 & 4 & 100 & \\
\hline Loudness & 0 & 0 & 3 & 75,0 & 5 & 55,6 & 0 & 0 & 0 & 0 & \\
\hline Não & 0 & 0 & 4 & 22,2 & 8 & 38,1 & 15 & 65,2 & 6 & 50,0 & \\
\hline Não lembra & 5 & 83,3 & 10 & 55,6 & 4 & 19,0 & 6 & 26,1 & 2 & 16,7 & \\
\hline Total & 6 & 100 & 18 & 100 & 21 & 100 & 23 & 100 & 12 & 100 & \\
\hline
\end{tabular}

Teste do Qui-quadrado; * Diferenças significantes $p<0,05$ 
Tabela 4. Distribuição numérica e percentual dos hábitos nocivos e benéficos à própria voz na opinião dos adolescentes $(n=80)$

\begin{tabular}{lllll}
\hline \multirow{2}{*}{ Hábitos vocais } & \multicolumn{2}{c}{ Sim } & \multicolumn{2}{c}{ Não } \\
\cline { 2 - 6 } & $\mathrm{N}$ & $\%$ & $\mathrm{~N}$ & $\%$ \\
\hline Hábitos nocivos & & & & \\
$\quad$ Gritar & 30 & 37,5 & 50 & 62,5 \\
$\quad$ Ingerir gelado & 26 & 32,5 & 54 & 67,5 \\
$\quad$ Tabagismo & 22 & 27,5 & 58 & 72,5 \\
\hline Hábitos benéficos & & & & \\
$\quad$ Beber água & 31 & 38,8 & 49 & 61,2 \\
$\quad$ Cantar & 30 & 37,5 & 50 & 62,5 \\
$\quad$ Não gritar & 20 & 25,0 & 60 & 75,0 \\
\hline
\end{tabular}

atribuídas características negativas, $6(27,3 \%)$ rapazes e 4 $(6,9 \%)$ moças citaram o pitch como uma característica negativa determinante para a sua voz e outras $4(6,9 \%)$ moças citaram a loudness; boa parte das adolescentes $(23-39,4 \%)$ relataram indiferença ao caracterizar a sua voz. Os parâmetros vocais, como a loudness e o pitch, foram fatores determinantes para descrever a voz desses adolescentes. Este fato corrobora a literatura, pois as principais características vocais relatadas pelos participantes ao descrever a voz na adolescência foram relacionadas ao pitch, à qualidade vocal e a loudness ${ }^{(5)}$.

No tópico referente à comparação da autopercepção da voz e a variável sexo (Tabela 2) apresentou uma diferença significante $(\mathrm{p}<0,001)$. Verificou-se que $10(45,5 \%)$ rapazes e $32(55,2 \%)$ moças referiram ter uma voz normal; os demais identificaram sua voz como desviada: $12(54,5 \%)$ rapazes e $6(10,3 \%)$ moças classificaram sua voz desviada quanto ao pitch, $18(31,0 \%)$ das adolescentes quanto à loudness e $2(3,5 \%)$ à qualidade vocal. Dentre os que classificaram suas vozes como desviadas, o pitch agudo, a loudness fraca e a qualidade vocal rouca foram as características mais mencionadas. Em pesquisa anterior ${ }^{(6)}$, ao levantar junto aos adolescentes termos descritivos que atribuíam a sua própria voz, destacaram que 75,8\% dos adolescentes manifestavam conceitos positivos a respeito de sua voz, em que o pitch foi um fator determinante para descrevê-la, principalmente para os rapazes. Em outra pesquisa ${ }^{(5)}$, os homens relataram características vocais relacionadas ao pitch (voz instável, desafinada, grossa, fina), à qualidade vocal (rouca, rachada, fanhosa) e a loudness (fraca).

Ao abordar a comparação entre o nível de satisfação vocal e a variável sexo (Tabela 2), houve uma diferença significante $(\mathrm{p}<0,001)$. A maioria dos sujeitos, $13(59,1 \%)$ rapazes e 52 $(89,6 \%)$ moças revelaram satisfação com a sua voz. Para 13 (100\%) rapazes e $40(76,9 \%)$ moças, a justificativa era devido ao fato da voz combinar com a personalidade, portanto, não havia desejo de modificá-la. Esse fato corrobora pesquisa encontrada na literatura, ao se verificar que a muda e imagem vocal estão fortemente relacionadas com a auto-imagem e percepção de si mesmo no meio em que se vive ${ }^{(8)}$.

Quanto à comparação entre as modificações vocais e a variável sexo (Tabela 2), houve uma diferença significante $(p<0,001)$. Observou-se que parte dos adolescentes, $12(54,5 \%)$ dos rapazes e $8(14,1 \%)$ das moças perceberam mudanças em suas vozes nos últimos anos, principalmente características relacionadas ao pitch e loudness; porém a maioria (27 - 46,5\%) das garotas não percebeu modificações ou (23 - 39,4\%) não se lembra ter ocorrido modificações com a sua voz nos últimos anos. Estes resultados confirmam a literatura: os rapazes perceberam mais e são mais acometidos por modificações vocais do que as moças, além de citarem mais termos negativos e, mais especificamente, relacionados à instabilidade e quebras da frequência vocal. Também perceberam que os sentimentos e comportamentos relacionados à alteração vocal durante a muda foram negativos: vergonha, irritabilidade, ansiedade, constrangimento, evitar falar, entre outros ${ }^{(5-6)}$.

No que diz respeito à comparação entre a autopercepção de adolescentes em relação à voz e à variável faixa etária (Tabela 3), observou-se que quanto maior a faixa etária, melhor é a opinião sobre a própria voz: $1(16,7 \%)$ adolescente de 10-11 anos, $9(50,0 \%)$ de 12-13 anos, $6(28,6 \%)$ de $14-15$ anos, $15(65,2 \%)$ de 16-17 anos e $10(83,3 \%)$ adolescentes de 18-19 anos atribuíram características positivas a sua voz $(\mathrm{p}<0,004)$. Em relação à autopercepção da voz, percebeu-se mais relatos de voz desviada na faixa etária de 12-13 anos, 7 $(38,9 \%)$ referiram voz desviada quanto ao pitch e $2(11,1 \%)$ desviada quanto a loudness; e de 14-15 anos, $5(23,8 \%)$ relataram voz desviada quanto ao pitch e $8(38,1 \%)$ desviada quanto a loudness $(\mathrm{p}<0,001)$. O mesmo ocorreu com o nível de satisfação vocal, no qual os adolescentes que mais relataram insatisfação com a sua voz foram da faixa etária de $12-13$ anos (6-33,3\%) e 14-15 anos (5 - 23,8\%). Por fim, os adolescentes $(9-42,9 \%)$ da faixa etária de $14-15$ anos foram os que mais perceberam modificações na voz nos últimos $\operatorname{anos}(\mathrm{p}<0,003)$.

Esse conjunto de dados, encontrados a partir da comparação da autopercepção sobre a voz do adolescente e a faixa etária, sugeriu a forte relação entre faixa etária, muda vocal e percepção/opinião sobre a voz. Sabe-se que durante a adolescência, por volta dos 13 aos 17 anos, o tom vocal masculino abaixa uma oitava, favorecendo uma voz com mudanças de frequência e quebras sonoras, fato que, para alguns adolescentes, pode ter sido significado como algo positivo, se os sintomas vocais são vistos como demonstração de maturidade, ou negativo, quando eles se sentem embaraçados. Assim, acredita-se que adolescentes que estão ou passaram por essa faixa etária percebem as modificações vocais e, até mesmo, modificam sua autopercepção vocal com mais frequência do que os mais jovens ${ }^{(5,9-10)}$.

Ao relatarem hábitos que fazem mal a sua voz (Tabela 4), os mais citados foram: gritar $(37,5 \%)$, ingerir líquido gelado $(32,5 \%)$, falar alto e tabagismo $(27,5 \%)$. Ressalta-se que o termo tabagismo foi empregado para abranger tanto o consumo de cigarro quanto estar na presença de um fumante. Estes dados vão ao encontro dos resultados de outra pesquisa ${ }^{(6)}$, cujos hábitos nocivos à voz mais citados antes de uma intervenção fonoaudiológica foram: gritar $(49,4 \%)$, falar muito $(29,8 \%)$, ingestão de líquido gelado $(25,6 \%)$, falar com esforço $(22,1 \%)$ e fumar $(15,7 \%)$. Estes achados demonstram que o adolescente identifica os fatores que desfavorecem a emissão vocal e, consequentemente, prejudicam a função social da voz.

Em relação aos hábitos benéficos para sua voz (Tabela 4), 
os mais citados foram: beber água $(38,8 \%)$, cantar $(37,5 \%)$ e não gritar (25\%). Dados semelhantes aos encontrados em pesquisa $^{(6)}$, na qual os hábitos benéficos mais citados foram: beber água e em temperatura ambiente $(46,1 \%)$, não gritar $(26,0 \%)$, não falar muito $(25,0 \%)$, evitar ingerir líquidos gelados $(12,5 \%)$ e realizar exercícios vocais $(10,6 \%)$.

Percebeu-se que a população em geral e neste estudo, mais especificamente, os adolescentes recebem uma informação distorcida de que beber água em abundância é benéfico para a voz, mas a literatura mostra que a ingestão de água não é algo que traz benefícios diretos para a voz ${ }^{(11-12)}$. Esta é uma questão em que os profissionais envolvidos nas campanhas voltadas à promoção da saúde vocal e à prevenção de problemas vocais devem ficar atentos.

Em linhas gerais, observou-se que a maioria dos adolescentes tem uma autopercepção em relação à própria voz, sendo algumas questões dependentes do sexo, da faixa etária, da forma que ele percebe sua voz e do meio em que ele vive.

\section{CONCLUSÃO}

A partir da análise dos resultados obtidos neste estudo, percebeu-se que quanto maior a faixa etária do adolescente, acima dos 16-17 anos, maior o seu nível de satisfação vocal e melhor a opinião sobre a própria voz. Em relação ao sexo, as adolescentes relatam um menor impacto na voz do que os rapazes, até mesmo devido às alterações fisiológicas mais acentuadas inerentes ao sexo masculino. Dessa forma, verifica-se que há uma forte relação existente entre a percepção/opinião sobre a voz, faixa etária e sexo, pois após toda a modificação fisiológica que marca essa fase, a voz é mais uma ferramenta que pode refletir seu ingresso na fase adulta.

Por fim, ressalta-se a importância do trabalho do fonoaudiólogo junto ao adolescente para que ele perceba que voz e comunicação, mesmo em transformação, interferem na sua interação com o outro, no seu ato de pensar, agir, fazer e na sua articulação com a realidade e a sociedade em que vive.

\begin{abstract}
Purpose: To verify the self-perception of adolescents concerning their own voice. Methods: A questionnaire regarding voice and communication was applied to 80 volunteer adolescents, 58 female (72.5\%) and 22 male (27.5\%), with ages ranging from 10 to 19 years, participants of the Program of Adolescent Healthcare of the State of São Paulo. Subjects answered the questionnaire themselves, and data were statistically analyzed. Results: The main age range was from 16 to 17 years $(23-28.8 \%)$. Half of the subjects (41 $51.3 \%$ ) reported positive vocal characteristics, classifying it as normal (42-52.5\%); in addition, $33(41.3 \%)$ did not detect vocal modifications during adolescence, demonstrating satisfaction with their production $(65-81.3 \%)$, referring that their voices matched their personality $(27-33.8 \%)$ and, therefore, they did not wish to modify it $(61-76,3 \%)$. The three most frequently reported negative vocal habits were: yelling $(30-37.5 \%)$, drinking cold beverages $(20-32.5 \%)$, and smoking $(22-27.5 \%)$. In turn, the subjects reported as beneficial vocal habits: drinking water $(31-38.8 \%)$, singing $(30-37.5 \%)$ and not yelling $(20-25 \%)$. Young men reported vocal problems related to pitch $(54.5 \%, \mathrm{p}<0.001)$, and young women, to loudness $(32.1 \%, \mathrm{p}<0.001)$. These problems were pointed out as impairing factors to their communication. Conclusion: The perception of adolescents about their voice and communication interferes in their relationship with others, in their way of thinking and acting, and the way they interact with reality and the society.
\end{abstract}

Keywords: Communication; Voice; Voice disorders; Adolescent; Self concept

\title{
REFERÊNCIAS
}

1. World Health Organization. Division of Reproductive Health.Technical Support. World Health Day. Safe motherhodd. 7 april 1998 Delay Childbearing (WHD 98.04) [Internet]. Geneva:WHO; 1998. [cited 2004 Mar 3]. Available from: URL: http://www.who.int/docstore/worldhealth-day/en/pages1998/whd98_04.html

2. Andrews ML, Summers AC. Voice therapy for adolescents. San Diego, Calif.: Singular Pub. Group; c1991.

3. Andrews ML. The singing/acting child: a speech-language pathologist's perspective. J Voice. 1997;11(2):130-4.

4. Kasama ST, Brasolotto AG. Percepção vocal e qualidade de vida. PróFono. 2007;19(1):19-28.

5. Gil D, Lourenço L, Miranda AR, Pereira AJ, Rodrigues S, Behlau M. A memória da muda vocal. Acta AWHO. 1994;13(2):74-80.

6. Almeida AAF, Ferreira LP. Cuidados com a voz: uma proposta de intervenção fonoaudiológica para adolescentes. Distúrb Comum. 2007;19(1):81-92.

7. Aggleton P. Trabalhando com jovens: implicações para a pesquisa e a organização de programas. Adolesc Latinoam. 2001;2(3):138-47.

8. Costa SA, Cruz OLM, Oliveira JAA. Otorrinolaringologia. Porto Alegre: Artes Médicas; 1994.

9. Boone DR, McFarlane SC. Voz e a terapia vocal. 5a ed. Porto Alegre: Artes Médicas; 1994.

10. Greene MCL. Distúrbios da voz. 4a. ed. São Paulo: Manole; 1989.

11. Verdolini-Marston K, Titze R, Druker DG. Changes in phonation threshold pressure with induced conditions of hydration. J Voice. 1990;4(2):142-51.

12. Verdolini K, Titze IR, Fennell A. Dependence of phonatory effort on hydration level. J Speech Hear Res. 1994;37(5):1001-7. 
Anexo 1. Protocolo de voz e comunicação direcionado a adolescentes

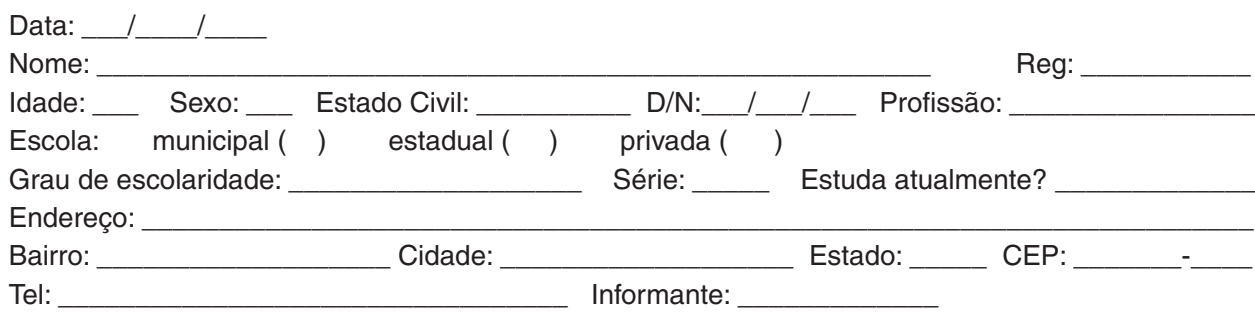

\section{I- Pesquisa da imagem vocal e da auto-imagem}

1) O que você acha da sua voz?

2) Você acha que sua voz é:
( ) rouca
( ) fraca
( ) fina
( )forte
( ) outros
( ) grossa
( ) normal

3) Você acha que sua voz combina com você?

( ) $\operatorname{sim}$ ( ) não

Por quê?

4) Você gostaria de mudar sua voz?

( ) $\operatorname{sim}$ ( ) não

Por quê?

Se sim, o que?

5) As pessoas comentam sobre a sua voz?

( ) $\operatorname{sim}($ ) não

Se sim, o que?

O que você acha disso?

6) Você percebeu alguma diferença na sua voz nos últimos anos?
( ) sim ( ) não ( ) não lembro
( ) não sei

Se sim, qual a diferença?

Como percebeu?

Como se sentiu?

7) Como se sente quando está falando (comentando) das coisas que pensa, faz e sente?

\section{II- Pesquisa de hábitos vocais}

8) Cite 3 coisas que você acha que faz BEM a sua voz:

9) Cite 3 coisas que você acha que faz MAL a sua voz:

\section{III- Pesquisa de aspectos da comunicação}

10) Você já ouviu falar algo sobre comunicação?

( ) $\operatorname{sim}($ ) não

O que?

11) Como você se comunica?

12) Você se comunica bem?

( ) $\operatorname{sim}($ ) não

Por quê?

13) Você tem dificuldade para se comunicar?

( ) $\operatorname{sim}($ ) não

Por quê?

14) De que maneira você se expressa melhor?

15) As pessoas conseguem entender (exatamente) o que você quer dizer? (se não é entendido(a), qual é o seu comportamento na maioria das vezes: anotar as particularidades mencionadas).

( ) sim ( ) não

Por quê? 